Article

\title{
SCRaMbLE: A Study of Its Robustness and Challenges through Enhancement of Hygromycin B Resistance in a Semi-Synthetic Yeast
}

\author{
Jun Yang Ong ${ }^{1,2,+}$, Reem Swidah ${ }^{1,+} \mathbb{D}$, Marco Monti ${ }^{1} \mathbb{D}$, Daniel Schindler ${ }^{1,3}\left(\mathbb{D}\right.$, Junbiao Dai ${ }^{4,5,6}$ \\ and Yizhi Cai $1,4,5,6, *$
}

Citation: Ong, J.Y.; Swidah, R.;

Monti, M.; Schindler, D.; Dai, J.; Cai, Y. SCRaMbLE: A Study of Its

Robustness and Challenges through Enhancement of Hygromycin B Resistance in a Semi-Synthetic Yeast. Bioengineering 2021, 8, 42. https:// doi.org/10.3390/bioengineering8030042

Academic Editor: Danièle Noël

Received: 2 March 2021

Accepted: 20 March 2021

Published: 23 March 2021

Publisher's Note: MDPI stays neutral with regard to jurisdictional claims in published maps and institutional affiliations.

Copyright: (C) 2021 by the authors. Licensee MDPI, Basel, Switzerland. This article is an open access article distributed under the terms and conditions of the Creative Commons Attribution (CC BY) license (https:/ / creativecommons.org/licenses/by/ $4.0 /)$.
1 Manchester Institute of Biotechnology (MIB), The University of Manchester, 131 Princess Street, Manchester M1 7DN, UK; j.y.ong@uu.nl (J.Y.O.); reem.swidah@manchester.ac.uk (R.S.); marco.monti@postgrad.manchester.ac.uk (M.M.); Daniel.Schindler@mpi-marburg.mpg.de (D.S.)

2 Department of Chemical Biology and Drug Discovery, Utrecht Institute for Pharmaceutical Sciences, and Bijvoet Center for Biomolecular Research, Utrecht University, Universiteitsweg 99, 3584 CG Utrecht, The Netherlands

3 Max-Planck Institute for Terrestrial Microbiology, Karl-von-Frisch-Straße 10, 35043 Marburg, Germany

4 CAS Key Laboratory of Quantitative Engineering Biology, Shenzhen Institutes of Advanced Technology, Chinese Academy of Sciences, Shenzhen 518055, China; junbiao.dai@siat.ac.cn

5 Guangdong Provincial Key Laboratory of Synthetic Genomics, Shenzhen Institutes of Advanced Technology, Chinese Academy of Sciences, Shenzhen 518055, China

6 Shenzhen Key Laboratory of Synthetic Genomics, Shenzhen Institutes of Advanced Technology, Chinese Academy of Sciences, Shenzhen 518055, China

* Correspondence: yizhi.cai@manchester.ac.uk

+ Equal contributors.

Abstract: Recent advances in synthetic genomics launched the ambitious goal of generating the first synthetic designer eukaryote, based on the model organism Saccharomyces cerevisiae (Sc2.0). Excitingly, the Sc2.0 project is now nearing its completion and SCRaMbLE, an accelerated evolution tool implemented by the integration of symmetrical loxP sites (loxPSym) downstream of almost every non-essential gene, is arguably the most applicable synthetic genome-wide alteration to date. The SCRaMbLE system offers the capability to perform rapid genome diversification, providing huge potential for targeted strain improvement. Here we describe how SCRaMbLE can evolve a semi-synthetic yeast strain housing the synthetic chromosome II (synII) to generate hygromycin B resistant genotypes. Exploiting long-read nanopore sequencing, we show that all structural variations are due to recombination between loxP sites, with no off-target effects. We also highlight a phenomenon imposed on SCRaMbLE termed "essential raft", where a fragment flanked by a pair of loxPSym sites can move within the genome but cannot be removed due to essentiality restrictions. Despite this, SCRaMbLE was able to explore the genomic space and produce alternative structural compositions that resulted in an increased hygromycin B resistance in the synII strain. We show that among the rearrangements generated via SCRaMbLE, deletions of YBR219C and YBR220C contribute to hygromycin B resistance phenotypes. However, the hygromycin B resistance provided by SCRaMbLEd genomes showed significant improvement when compared to corresponding single deletions, demonstrating the importance of the complex structural variations generated by SCRaMbLE to improve hygromycin B resistance. We anticipate that SCRaMbLE and its successors will be an invaluable tool to predict and evaluate the emergence of antibiotic resistance in yeast.

Keywords: Saccharomyces cerevisiae; synthetic yeast; SCRaMbLE; hygromycin B; accelerated evolution; Sc2.0

\section{Introduction}

In the many years of scientific studies to understand the fundamentals of life, molecular biology has seen many breakthroughs, however much mystery still remains. Is our 
current understanding of biological systems sufficient to create and improve on nature? In 2008, we saw the first complete synthesis of the blueprint of life-the $582 \mathrm{~kb}$ genome of the bacteria Mycoplasma genitalium [1]. Now, the synthesis of an entire eukaryotic genome is on the horizon: a designer version of the $12 \mathrm{Mb}$ genome of Saccharomyces cerevisiae, also known as baker's yeast. This time around, the goal has been not only to synthesise the complete genome, but also to incorporate novel design features [2].

Several rational design principles form the basis of the Sc2.0 genome [2]. One of the most prominent changes is the implementation of SCRaMbLE. The synthetic chromosome recombination and modification by LoxP-mediated evolution (SCRaMbLE) system is initiated by the introduction of symmetrical loxP sites (loxPsym) 3 bp downstream of most nonessential genes. This allows massive genomic rearrangements upon induction of the Cre recombinase, creating a pool of highly diverse genotypes. The application of SCRaMbLE has proven its power to generate genomic diversity through condition-specific strain evolutions [3,4]. These have led to enhanced growth on an alternative carbon source (xylose) [5], improved production from heterologous pathways for compounds such as violacein [5,6], penicillin, carotenoids [6,7] and betulinic acid [8], and increased resistance to alkaline conditions, high temperature, ethanol and acetic acid $[9,10]$.

Given its potential, we anticipate that SCRaMbLE could be a powerful tool to combat a current threat to the health of humankind-the emergence of antibiotic resistance in pathogens. Despite the gravity of our current situation, the discovery of new antibiotics is a painfully slow process. We must therefore anticipate and prepare for antibiotic resistance early if we hope to stop it from happening, or to have the solution ready for when it occurs. Selection for resistance happens naturally in microbes over generations. However, accelerated evolution offered by technologies such as SCRaMbLE allows us to expedite the process and to anticipate possible future emergence of antibiotic resistance in pathogens. Among all pathogenic infections, fungal infections kill more than 1.5 million and affect over a billion of people every year [11]. Taking the generally recognised as safe (GRAS) $S$. cerevisiae yeast as an example of fungi and a widely used laboratory antibiotic hygromycin $\mathrm{B}$, we use SCRaMbLE to evolve and deconvolute novel genotypes leading to hygromycin B resistance.

The choice of antibiotic in this study, hygromycin B, is an aminoglycoside antibiotic produced by Streptomyces hygroscopicus that was first isolated in 1958 [12]. Active against both prokaryotes and eukaryotes, hygromycin B antibiotic activity is attributed to the inhibition of protein synthesis [13] as it binds to RNA helix 44 (h44) of the small (30S) ribosomal subunit, next to the aminoacyl-tRNA binding (A) site [14-16]. Hygromycin B weakly stabilises the A site, but its potency results primarily from its inhibition of mRNA and tRNA translocation by blocking the tRNA path between the A and peptidyl-tRNA (P) sites $[13,17]$.

Some bacteria have developed resistance mechanisms towards hygromycin B. For example, in S. hygroscopicus itself, the hyg gene codes for hygromycin B 7"-O-kinase (HYG; EC 2.7.1.119), which phosphorylates hygromycin B at the 7"-hydroxyl group of the destomic acid ring [18-20]. In E. coli, hph codes for hygromycin B 4-O-kinase (HPH; EC 2.7.1.163), which phosphorylates hygromycin B at the 4-hydroxyl group of the 2-DOS ring [21]. hyg and $h p h$ have no homology [19], and they phosphorylate hygromycin B at different sites. The phosphorylated hygromycin B products are inactive and do not affect protein synthesis [19]. Alternatively, different point mutations in ribosomal RNA genes have been identified in prokaryotic and eukaryotic cells that provide resistance to hygromycin B, i.e., U1406C, C1496U and U1498C in 16S rRNA in Mycobacterium smegmatis [22], mutation of G1491 or C1409 in 16S rRNA in E. coli [23], and U1711C in 17S rRNA in Tetrahymena thermophila [24]. In these cases, resistance to hygromycin B may have arisen from subtle changes in secondary structure of rRNA leading to weakened interaction with hygromycin B.

The $h p h$ resistance gene from $E$. coli has been used as a selectable marker in eukaryotic cells including yeast $[25,26]$. Very few other genes linked to hygromycin B resistance have been described in yeast [27-29]. In this study we employed SCRaMbLE to accelerate the 
evolution of a semi-synthetic yeast strain (synII), which harbours the $770 \mathrm{~kb}$ synthetic chromosome II [30], in the presence of hygromycin B. This allowed us to introduce stochastic genome rearrangements on chromosome II, including deletion, inversion, duplication and translocation, to identify novel gene rearrangements which may improve yeast's resistance towards the antibiotic hygromycin B. Furthermore, we aim to gain insights into the capacity of the existing SCRaMbLE system to drive genotypic and phenotypic evolution by inspecting its capability to generate gene deletions which have been previously reported to increase hygromycin B resistance in yeast.

\section{Results and Discussion}

\subsection{Generating a Hygromycin B Resistant Strain Using SCRaMbLE}

To investigate whether using SCRaMbLE with the synII strain can improve its hygromycin B resistance, the parental synII strain (YCy1188) and the wild type strain (BY4742) were initially benchmarked for their resistance towards hygromycin B. Hygromycin B is usually used at the final concentration of $200 \mu \mathrm{g} / \mathrm{mL}$ for selection in yeast with the $h p h$ selectable marker $[25,26]$. A hygromycin B serial dilution spot assay was carried out using four biological replicates for each strain. For both BY4742 and synII, the lethal concentration was identified to be $150 \mu \mathrm{g} / \mathrm{mL}$ of hygromycin B after 2 days (Figure S1). Interestingly, synII was observed to show a slightly higher resistance to hygromycin B than BY4742, as shown by their differential growth at $150 \mu \mathrm{g} / \mathrm{mL}$ after 6 days of incubation. Our hypothesis is that the apparent increased resistance is due to the deletion of tRNA genes in synII, which has been reported to cause an up-regulation of translational machineries in the synII strain [30]. The up-regulation may have mitigated the binding of hygromycin B to the ribosomes subunit and therefore lessened the inhibition of protein synthesis. Similar up-regulation of the translational machinery was also observed in a Leishmania donovani strain resistant to paromomycin, another aminoglycoside antibiotic [31].

The SCRaMbLE experiment workflow is depicted in Figure 1. The Cre recombinase expression plasmid, pSCW11-Cre-EBD [32] was transformed into the synII strain (YCy2918) [3]. SCRaMbLE was induced for $24 \mathrm{~h}$ at $30^{\circ} \mathrm{C}$ with $1 \mu \mathrm{M}$ ß-estradiol in $10 \mathrm{~mL}$ selective SCD-His liquid media in order to maintain the pSCW11-Cre-EBD vector. Subsequently, the cells and the respective control (YCy2917 and YCy2919) were back-diluted to $0.1 \mathrm{OD}_{600}$ and plated onto the YPD plates containing $200 \mu \mathrm{g} / \mathrm{mL}$ of hygromycin $\mathrm{B}$. This was followed by $3-6$ days of incubation at $30{ }^{\circ} \mathrm{C}$. Thirty-three hygromycin B resistant SCRaMbLEd strains which grew successfully at the concentration of $200 \mu \mathrm{g} / \mathrm{mL}$ of hygromycin $\mathrm{B}$, were isolated and submitted for further characterisation. These strains were picked in the order of decreasing colony size as a tentative measure of their resistance. They were termed HYG2.1 to HYG2.33.

The first ten of the 33 SCRaMbLEd strains, HYG2.1 to HYG2.10, were characterised in depth for their hygromycin B resistance via a spot test (Figure 2). HYG2.1 (YCy4021) showed the highest resistance level among the ten SCRaMbLEd strains tested, being able to grow at $250 \mu \mathrm{g} / \mathrm{mL}$ hygromycin B, outperforming the parental strain by $40 \%$. 


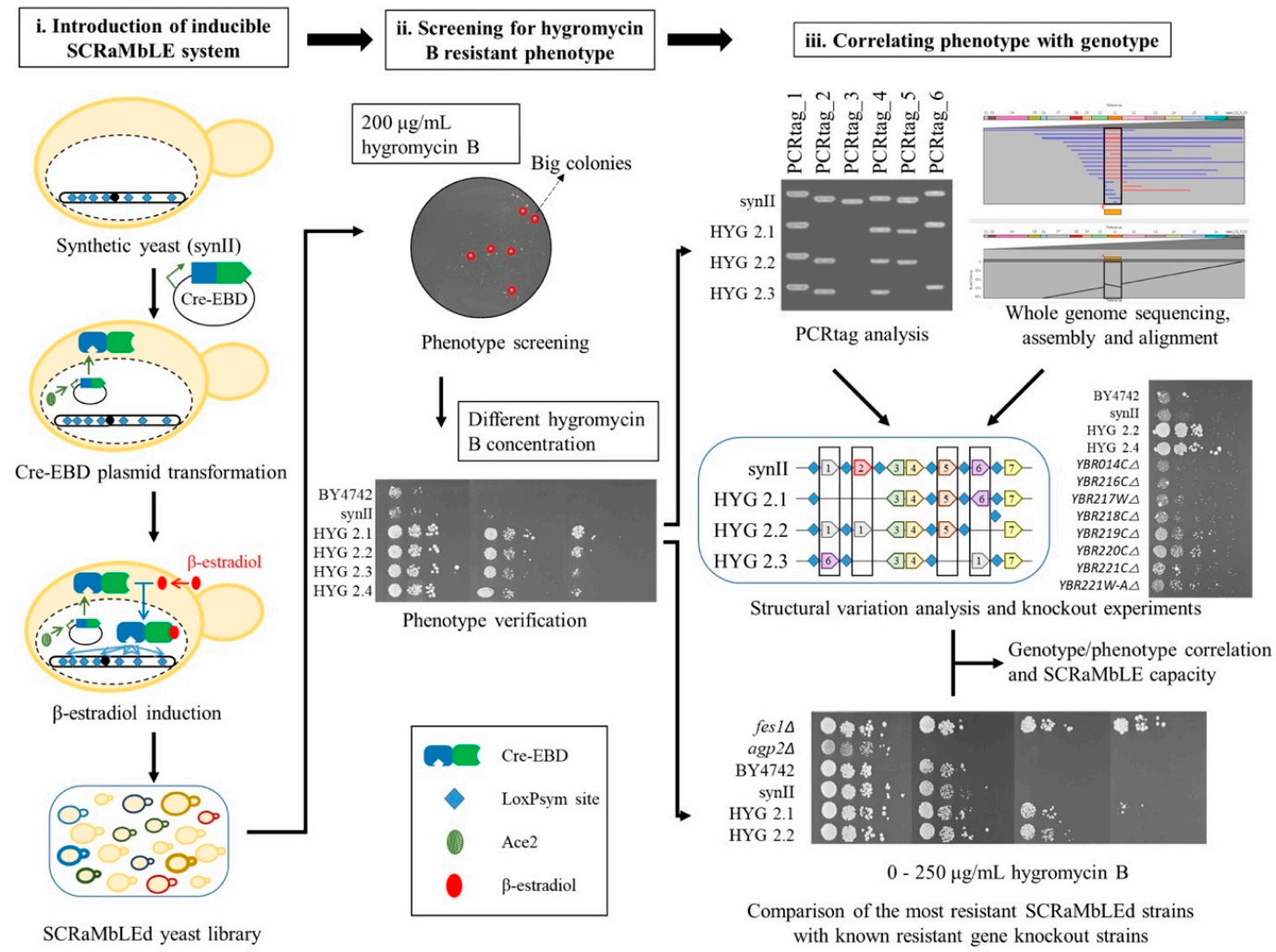

Figure 1. Schematics of a SCRaMbLE workflow to generate synthetic yeast with increased hygromycin B resistance. The workflow consists of three phases: (i) Introduction of the inducible SCRaMbLE system. The synthetic yeast is transformed with a pSCW11-Cre-EBD plasmid, expression of which is induced by estradiol to generate a library of SCRaMbLEd yeast strains; (ii) Screening for SCRaMbLEd yeast strains with hygromycin B resistant phenotype. SCRaMbLEd strains are first spread onto plates containing $200 \mu \mathrm{g} / \mathrm{mL}$ hygromycin B concentration to select for resistant strains, phenotypes of which are then verified by spot test analysis on different hygromycin B concentrations; (iii) Identifying links between the genotype and the resistance phenotype, and inspecting the capacity of SCRaMbLE. The resistant strains are subjected to PCRtag analysis and whole genome sequencing to identify any structural variation that may have contributed to the resistance phenotype. Individual knockout strains derived from the identified variations were tested to identify the contribution of each gene to the resistance phenotype. On the other hand, knockout strains with higher resistance based on literature are compared to those improved strains obtained through SCRaMbLE to see whether the best possible strain has been generated.

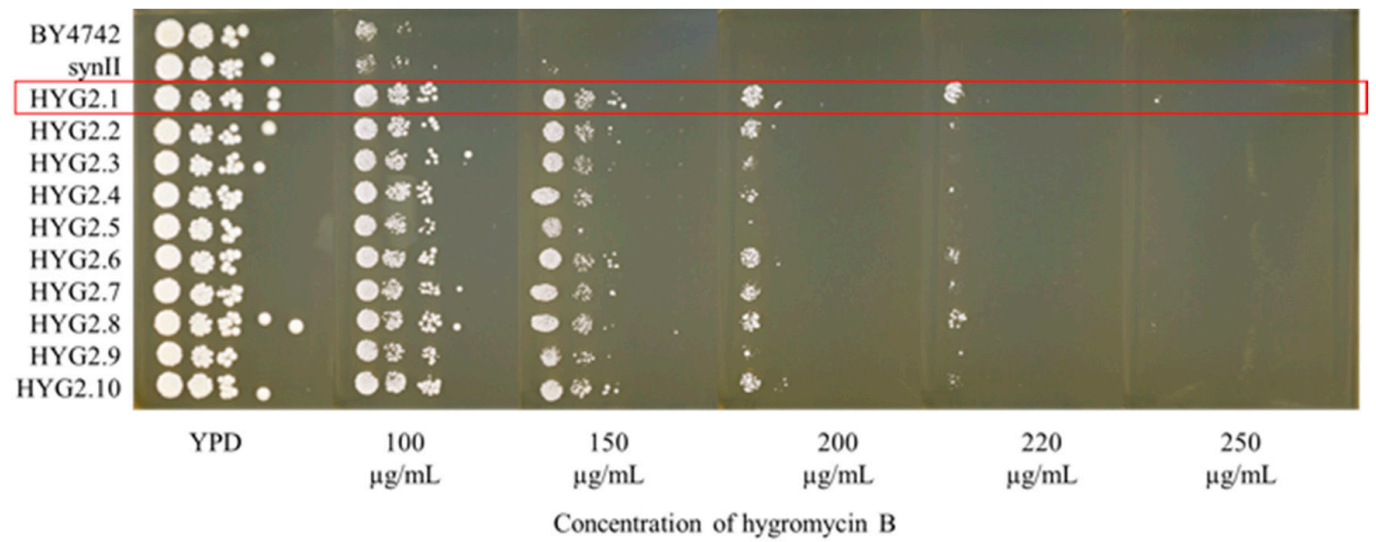

Figure 2. Spot test to assess hygromycin B resistance of the SCRaMbLEd strains HYG2.1-HYG2.10. SCRaMbLEd strains which grew at $200 \mu \mathrm{g} / \mathrm{mL}$ hygromycin B concentration were isolated and spotted on YPD plates containing different concentrations of hygromycin B. The plates were incubated at $30^{\circ} \mathrm{C}$ and the photos were taken on day 5 . SCRaMbLEd yeast strain HYG2.1 shows improved growth at higher hygromycin B concentrations compared to the wild type and the parental synII strains. 


\subsection{PCRTag Analysis for SCRaMbLEd Hygromycin B Resistant Strains}

Following SCRaMbLE, we carried out quick genotyping to identify gene deletions in five resistant SCRaMbLEd strains, namely HYG2.1, HYG2.2, HYG2.3, HYG2.4 and HYG2.5 (YCy4021, YCy4022, YCy4023, YCy4024, YCy4025) (Figure S2) using PCRTag analysis.

PCRTag is a barcode system incorporated into Sc2.0 chromosomes by synonymous codon recoding of two $\sim 20$ bp regions, separated by around $500 \mathrm{bp}$, within each open reading frame (ORF) [2]. This allows us to distinguish between synthetic and wild type sequences via a simple PCR setup using primers for either the synthetic or the wild type allele. Furthermore, as applied here, PCRTag analysis allows us to detect the presence or absence of each synthetic ORF after SCRaMbLE.

Two gene deletions IML3 (YBR107C) and AIM3 (YBR108W) were detected in HYG2.1 ( $c f$. DEL-1), while a deletion of the gene GRX7 (YBR014C) was detected in HYG2.2 (cf. DEL5). Multiple deletions were detected in HYG2.2 and HYG2.4 strains and summarized in Supplementary Table S1. All of these deletions were confirmed by nanopore sequencing (see Section 2.3).

The PCRTag analysis is only able to indicate the presence or absence of a gene. PCRTag analysis cannot therefore identify inversions, duplications or translocations, which are other possible events resulting from SCRaMbLE. In this case, a more thorough analysis of the structural variations in the SCRaMbLEd strains is made possible by genome sequencing.

\subsection{Nanopore Sequencing Analysis for Hygromycin B Resistant Strains}

Short read sequencing has been shown to be inadequate for solving complex structural variations in SCRaMbLEd genomes [4]. However, third generation sequencing techniques, such as nanopore sequencing, provide ultralong reads that can be used to solve highly complex structural variations in SCRaMbLEd yeast [5]. We therefore decided to analyse the genomes of three strains with the highest hygromycin B resistance (HYG2.1, HYG2.2 and HYG2.4) via nanopore sequencing.

Our Nanopore sequencing result has a coverage from 7.15 to 13.23 -fold with a mean read length of $16 \mathrm{~kb}$ and an N50 of $36 \mathrm{~kb}$. The longest obtained read which mapped to the genome was $171 \mathrm{~kb}$. An overview of the sequencing data is available in (Figure 3D).

Interestingly, the sequencing data indicated an additional copy of TSC10 (YBR265W) in chromosome 8 (INS-0) in all SCRaMbLEd isolates as well as in the parental synII strain (YCy1188). This was found to be the result of an off-target integration of the wild type TSC10-URA3 at ARS810 in chromosome 8. The wild type TSC10-URA3 integration was intended to replace the synthetic YBR265W gene in synII (YCy1189) as it was identified as the cause of a growth defect [30]. Our sequencing data showed that homologous replacement did not take place but wild type TSC10 was integrated as an additional copy on chromosome 8 (Figure S3).

All other structural variations detected were between loxPsym sites, confirming that structural variations were caused by Cre recombinase. Supplementary Table S1 highlights all detected structural variations within the sequenced strains, where each structural variation (INS = insertion, DEL = deletion, INV = inversion, DUP = duplication, INVDUP $=$ inverted duplication) is accompanied by a number $(0,1,2$, etc., in the order mentioned) for cross-reference on the dot plot (Figure 3).

The strain HGY2.1 (YCy2934) contains a single deletion (DEL-1) of approximately $4.5 \mathrm{~kb}$ carrying both IML3 (YBR107C) and AIM3 (YBR108W) genes. Iml3 plays a role in kinetochore function and a null mutation leads to defects in the segregation of chromosomes and minichromosomes [33]. The Aim 3 protein works together with Abp1 to inhibit barbedend actin filament elongation [34]. Neither of these genes have been associated with increased resistance to hygromycin B in the WT background strain, or other aminoglycoside antibiotics through systematic mutation sets, and their functions indicate no obvious contribution to hygromycin B resistance. However, it is possible that deletions of IML3 (YBR107C) and AIM3 (YBR108W) in the synII strain could have a synergistic effect and associate somehow with the resistance phenotype in the synthetic background strain. 
$\mathbf{A}$

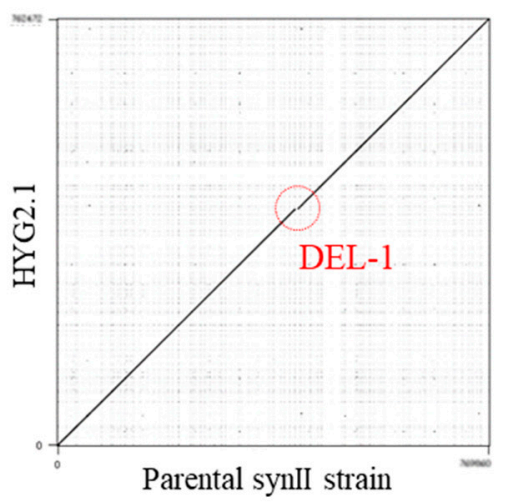

B

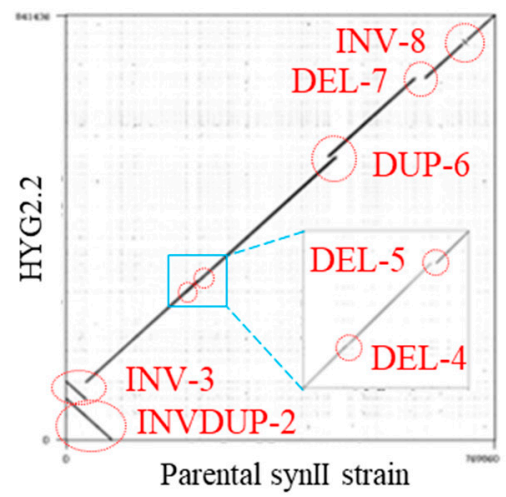

C

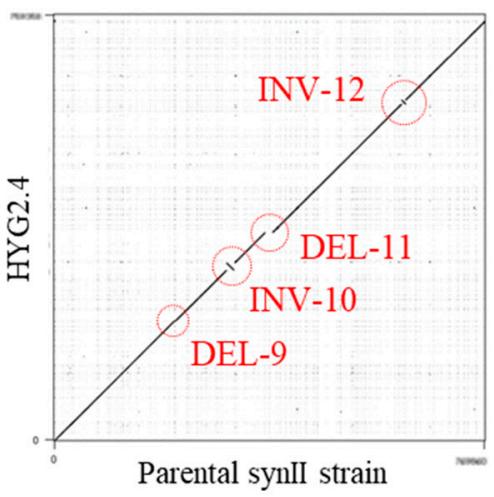

D

\begin{tabular}{|l|l|l|l|l|}
\hline & YCy1188 & HYG2.1 & HYG2.2 & HYG2.4 \\
\hline Mean read length (bp): & 16915.6 & 18179.4 & 16870.1 & 11441.2 \\
\hline Mean read quality: & 12.6 & 12.6 & 12.5 & 9.3 \\
\hline Median read length (bp): & 7741.0 & 10842.0 & 8735.0 & 3976.5 \\
\hline Median read quality: & 13.0 & 13.0 & 13.0 & 10.2 \\
\hline Number of reads: & 7569 & 4759 & 9489 & 10996 \\
\hline Read length N50 (bp): & 39153 & 35471 & 36073 & 32659 \\
\hline Total bases (bp): & $12.8 \times 10^{7}$ & $8.7 \times 10^{7}$ & $16.0 \times 10^{7}$ & $12.6 \times 10^{7}$ \\
\hline Coverage: & 10.58 & 7.15 & 13.23 & 10.40 \\
\hline
\end{tabular}

Figure 3. Dot plots illustrating the structural variations observed in the SCRaMbLEd strains HYG2.1 (A), HYG2.2 (B) and HYG2.4 (C) via nanopore sequencing. Each SCRaMbLEd strain (y-axis) is plotted against the parental synII (YCy1188) strain (x-axis) and each structural variation can be referenced to Supplementary Table S1. (D) Nanopore sequencing run data.

Strain HYG2.2 (YCy2935) and HYG2.4 (YCy2937) showed more complex variations, with in total eleven SCRaMbLE events between them. In HYG2.2 strain, we solved a complex structural variation consisting of a $79 \mathrm{~kb}$ duplication and inversion event (INVDUP-2), alongside an inversion of $34 \mathrm{~kb}$ in the same region (INV-3). DEL-4 is an additional $1 \mathrm{~kb}$ deletion which does not contain an ORF. Furthermore HYG2.2 contains a $1.3 \mathrm{~kb}$ deletion encoding GRX7 (DEL-5) and a $14 \mathrm{~kb}$ duplication (DUP-6). Finally, a $7 \mathrm{~kb}$ sequence encoding for BIT2, EFM2 and HSM3 is inverted (INV-8).

Strain HYG2.4 (YCy2937) shows evidence of four SCRaMbLE events, including one $2 \mathrm{~kb}$ centromeric deletion containing ARS208 and YBL001C (DEL-9). However, the area surrounding the centromere was duplicated but the centromere was deleted in one of the duplicated segments. Furthermore, we found a $13 \mathrm{~kb}$ inversion containing seven genes (YBR050C, YBR051W, YBR052C, YBR053C, YBR054W, YBR055C, YBR056W) (INV-10), a deletion of $14 \mathrm{~kb}$ (DEL-11) which was not detected by PCRTag analysis, and a $7 \mathrm{~kb}$ inversion containing four genes (INV-12).

The identified gene deletions presented an opportunity to match the variations in genotype with the observed resistance phenotype.

Accordingly, we proceeded to evaluate the impact of the identified gene deletions towards hygromycin B resistance in a WT background as the single knockout $(\mathrm{KO})$ yeast collection [35] is available resources in the lab for further characterization study in the lab and synII strain was originated from BY4741. A total of 22 corresponding single deletion strains were selected from the single knockout $(\mathrm{KO})$ yeast collection [35] for spot test analysis on YPD containing different concentrations of hygromycin B (Figure 4). Surprisingly, both $i m l 3 \Delta$ and aim $3 \Delta$ single $\mathrm{KO}$ strains exhibited a sensitive phenotype towards 
hygromycin B compared to BY4742, synII and HYG2.1. This suggests that the resistance phenotype of HYG2.1 generated during SCRaMbLE was not caused by either IML3 or AIM3 deletion alone. Interestingly, we observed small but noticeable improvements in hygromycin B resistance in single knockout strains of two uncharacterized genes YBR219C, $Y B R 220 C$. In addition, slight hygromycin B resistance improvement was associated with $Y B R 084 C-A$ deletion. Effects of these gene deletions on hygromycin $B$ resistance have not been reported before.

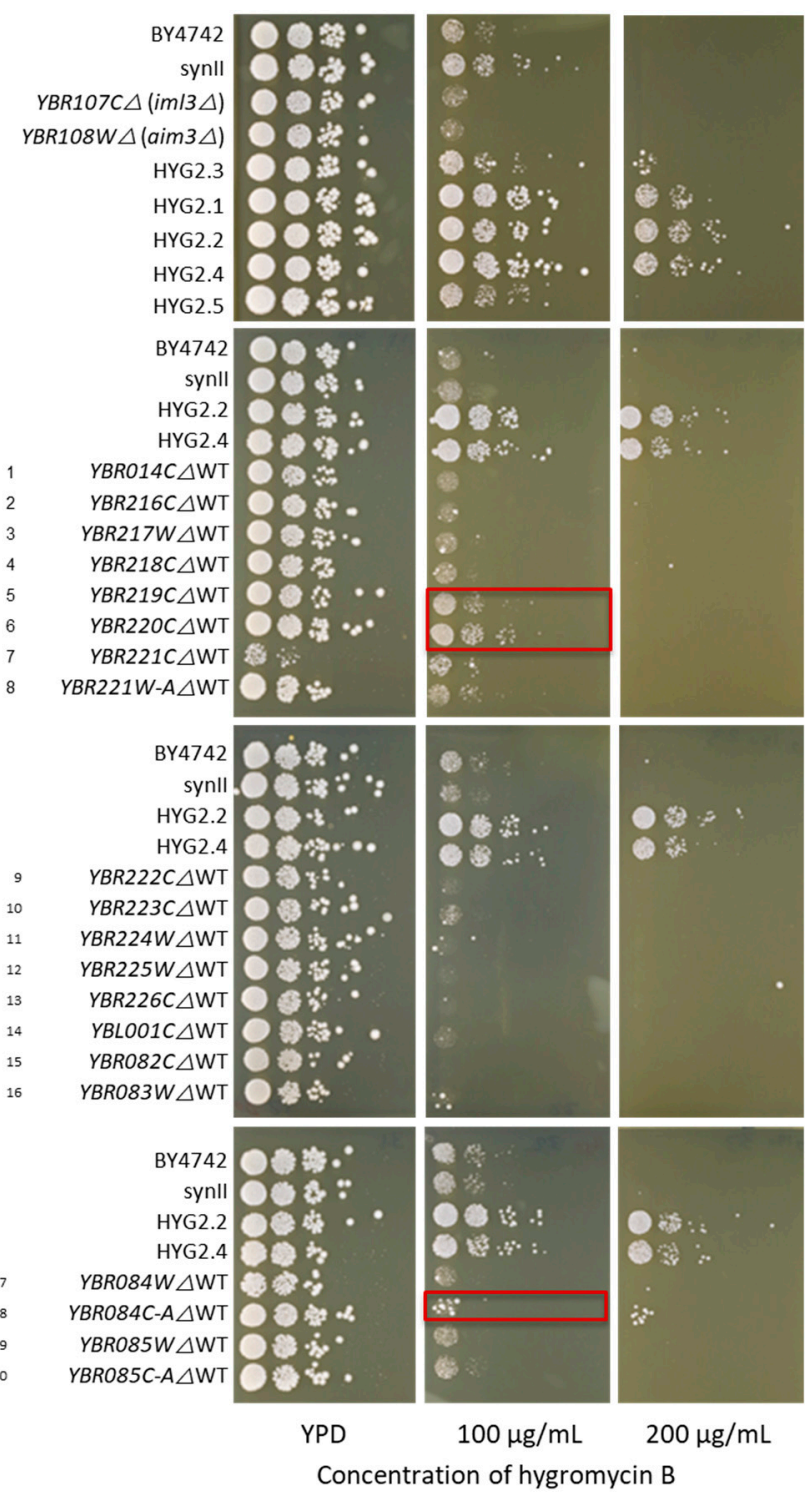

Figure 4. Phenotypic analysis showed that $Y B R 219 C \Delta W T, Y B R 220 C \Delta W T$ and $Y B R 084 C-A \Delta W T$ contributed to hygromycin B resistance. Single knockout strains, BY4742, synII, SCRaMbLEd strains were spotted on YPD plates containing different concentrations of hygromycin B. Plates were incubated at $30^{\circ} \mathrm{C}$ and the photos were taken on day 3 . The increased of hygromycin B resistance is slightly increased when YBR219C $\triangle \mathrm{WT}, Y B R 220 C \Delta \mathrm{WT}$ and $Y B R 084 C-A \Delta \mathrm{WT}$ were deleted individually from the WT strain. The hygromycin B resistance is significantly higher in the SCRaMbLEd strains HYG2.2 and HYG2.4 compared to single deletion strains.

None of the single deletion strains were shown to be able to compete with the resistance level shown by the SCRaMbLEd strains harbouring complex, combinatorial rearrangements. This demonstrates the power of SCRaMbLE to explore complex structural variations beyond single deletions to rapidly produce desired phenotypes. 
For this reason, we investigated whether combining the observed deletions in a double knockout strain could reproduce the observed hygB resistance phenotype. We decided to generate two double deletion mutants based on our single knockout strain analysis. We hypothesized that these deletions are the major contributing factors for the observed phenotype of HYG2.1 and HYG2.4. HYG2.1 contains the deletion of YBR107C and YBR108W. The individual deletions do not improve the phenotype (Figure 4). HYG2.4 accumulated multiple deletions but in the single knockout analysis only YBR219CA and YBR220CA slightly improve the hygB resistance phenotype (Figure 4). Therefore, the double deletion strains YBR107C $\triangle$ YBR108WD and YBR219CD YBR220CD strains were constructed in the BY4742 or BY4741 starting from single strains out of the knockout (KO) yeast collection [35]. The double deletion strain YBR107C $\triangle$ YBR108WD indicates a slight improvement compared to the single knockout strains $Y B R 107 C \Delta$ and $Y B R 108 W \Delta$ on YPD supplemented with $100 \mu \mathrm{g} / \mathrm{mL}$ hygromycin B. However, the level of the hygB resistance improvement was less compared to HYG2.1. In contrast, the double knockout YBR219CA YBR220CA strain has a severe growth defect even under standard growth conditions and does not improve the resistance phenotype towards hygB (Figure 5). It is more likely the resistance phenotype in the SCRaMbLEd strains is related to the complex rearrangements generated via SCRaMbLE which play a synergistic effect towards hygB resistance phenotype. Future transcriptome and proteome analysis may shed light into the complex situation causing the observed phenotype. Potentially, the effects might be accentuated by or particular to the synthetic yeast background.

A

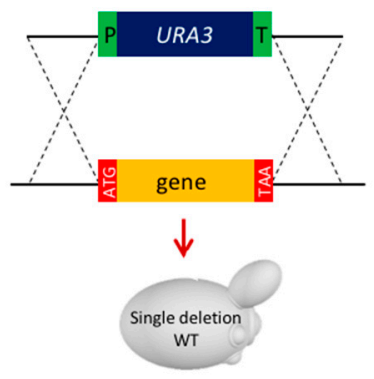

B

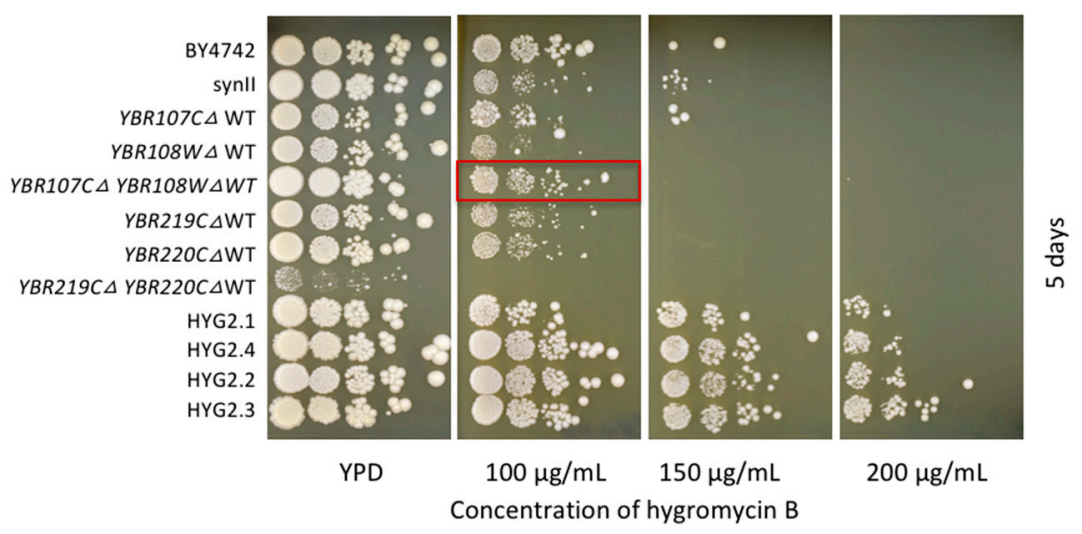

Figure 5. Phenotypic analysis showed that SCRaMbLEd strains are more resistant to hygromycin B compared to single/double deletions strains. (A) Visualization of the applied deletion strategy using the endogenous homologous recombination machinery. (B) Spot test. BY4742, synII, single knockout, double knockout and SCRaMbLEd strains were spotted on YPD plates containing different concentrations of hygromycin B. Plates were incubated at $30^{\circ} \mathrm{C}$ and the photos were taken on day 5. The double deletion strain YBR107C $\triangle$ YBR108W $\mathrm{WT}$ grows slightly better compared to the single deletion $Y B R 107 C \Delta$ and $Y B R 108 W \Delta$ strains on the YPD plate $+100 \mu \mathrm{g} / \mathrm{mL}$ hygromycin $\mathrm{B}$. Interestingly, YBR219CA. YBR220CAWT strain has a severe growth defect on the normal growth conditions and it does not improve the resistance phenotype toward hygromycin $\mathrm{B}$. 


\subsection{Yeast Knockout Library Assessment of Hygromycin B Resistance}

Whilst SCRaMbLE successfully produced strains with improved hygromycin B resistance, we sought to inspect the capability of SCRaMbLE to generate the optimal genomic variations for this trait. We assessed whether the variations generated by SCRaMbLE mentioned above could outcompete known hygromycin B resistant strains based on large-scale screening of the YKO collection. In chromosome 2, the deletion of NCL1 (YBL024W) [28], ECM8 (YBR076W) [29], FES1 (YBR101C) [28] and AGP2 (YBR132C) [27] were previously reported to cause a hygromycin $B$ resistant phenotype. For the purpose of this study, two single knockout strains were selected as positive controls for hygromycin B resistance, fes1 $\Delta:: k a n M X 6$ (YCy2983) and agp2 $\Delta:: k a n M X 6$ (YCy2984) [35].

fes1 $\Delta$ and agp $2 \Delta$ were compared to the resistant SCRaMbLEd strains HYG2.1-HYG2.5 (Figure 6A). fes1 $\Delta$ strain showed a noticeably higher resistance to hygromycin B compared to BY4742 and synII, in agreement with the previous report [28], as well as compared to SCRaMbLEd strains HYG2.1- HYG2.5. Unexpectedly, the agp2 $\Delta$ strain showed high sensitivity to hygromycin $\mathrm{B}$, in contrast to what was previously reported [27].

\section{A}

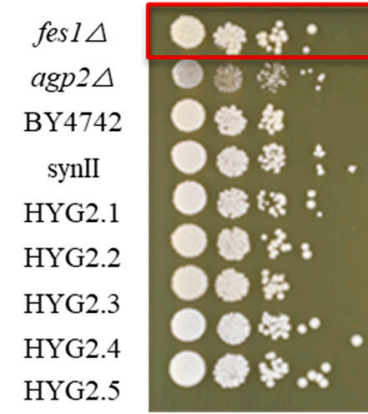

YPD

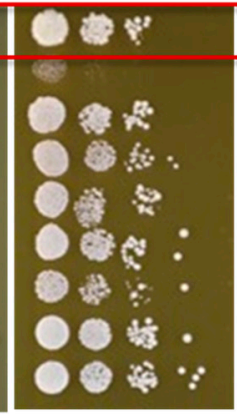

$100 \mu \mathrm{g} / \mathrm{ml}$

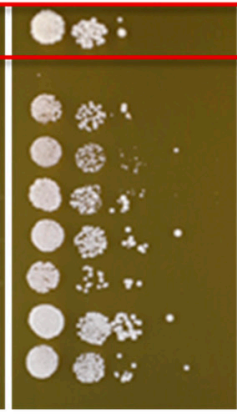

$150 \mu \mathrm{g} / \mathrm{ml}$

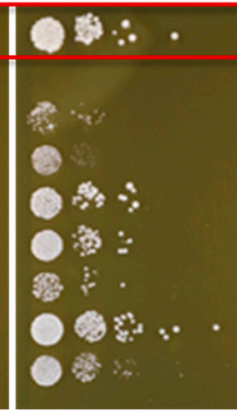

$180 \mu \mathrm{g} / \mathrm{ml}$

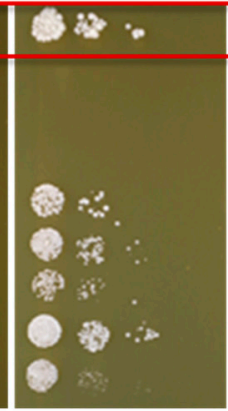

$200 \mu \mathrm{g} / \mathrm{ml}$



$250 \mu \mathrm{g} / \mathrm{ml}$

Concentration of hygromycin B

B

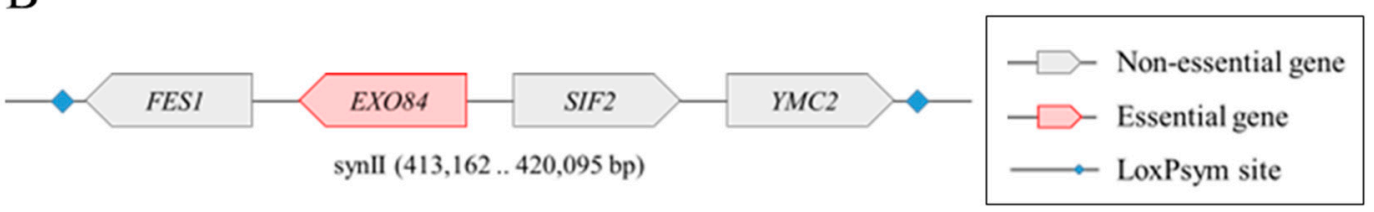

Figure 6. (A) Phenotypic analysis revealed that fes $1 \Delta$ and agp $2 \Delta$ had contrary effect on the hygromycin B resistance. Single knockout strains fes1 $\Delta$ and agp2 $\Delta$, BY4742, synII, SCRaMbLEd strains HYG2.1-HYG2.5 were spotted on YPD plates containing different concentrations of hygromycin B. Plates were incubated at $30^{\circ} \mathrm{C}$ and the photos were taken on day 3 . fes1 $\Delta$ increased the resistance towards hygromycin B [28], while agp2 $\Delta$ led to sensitivity to hygromycin B in contrast to what was reported [27]. (B) The loxPsym sites flanking FES1 enclose 3 other genes, EXO84, SIF2 and YMC2, in which EXO84 is an essential gene.

Fes1 is an Hsp70 nucleotide exchange factor, essential for the degradation of misfolded proteins by the ubiquitin-proteasome system. fes $1 \Delta$ has been associated with an unusually strong and constitutive heat shock response at $25^{\circ} \mathrm{C}[36,37]$. fes $1 \Delta$ has also been reported to cause bleomycin resistance in S. cerevisiae [38]. However, the mechanism via which fes $1 \Delta$ causes resistance towards bleomycin or hygroymycin B is unclear.

We explored why FES1 deletion was not detected in SCRaMbLEd strains despite the exceptional capacity of SCRaMbLE to generate other structural variations leading to higher hygromycin B resistance, albeit those less effective than fes1 deletion itself. Scrutiny of the synII chromosome map revealed that during SCRaMbLE, FES1 is linked to an essential 
gene. The loxPsym sites flanking FES1 enclose three other genes: SIF2, YMC2 and the essential EXO84 (Figure 6B). This is due to the Sc2.0 design principle where loxPsym sites are only located $3 \mathrm{bp}$ downstream of non-essential genes.

We termed this connection of multiple essential/non-essential genes in a single loxP flanked unit an "essential raft", where the affected genes can move together but cannot be deleted from the synthetic genome. This highlights a limitation in the ability of the SCRaMbLE system.

Despite the drawbacks, we recognise this limitation as an opportunity for our research goal. The generation of a single deletion would already be possible with other techniques, while SCRaMbLE is better poised for more complex gene rearrangements. It was without the domination of FES1 deletion in the SCRaMbLEd strains that allowed us to identify other structural variations that led to increased resistance and have not been reported before.

Despite the undesired effect associated with the fes 1 essential raft, we attempted to further increase hygromycin B resistance by carrying out additional rounds of SCRaMbLE on HYG2.1, the strain that demonstrated the one of the highest resistances after the first round of SCRaMbLE. However, no candidates with further improvement of hygromycin B resistance were obtained from two biological replicates. As a result, we reasoned that the first round of SCRaMbLE in this genotype background was already sufficient to maximise hygromycin B resistance in the context of a single synthetic chromosome II. This may have reached the local minimal in the genetic space, or alternatively the additional genomic rearrangements from further SCRaMbLE rounds may have resulted in reduced fitness in descendent strains, a phenomenon also described previously by Blount and coworkers [5].

\section{Materials and Methods}

\subsection{Strains and Media}

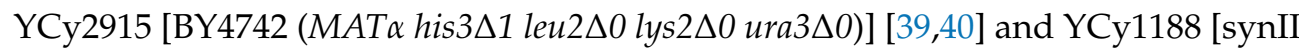

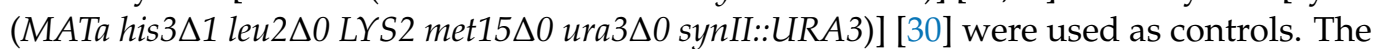
pSCW11-cre-EBD plasmid was introduced into SynII and used for the induction of SCRaMbLE. All SCRaMbLEd strains generated in this study are listed in Supplementary Table S2. Strains were grown on either standard yeast extract/peptone/dextrose media (YPD) for non auxotrophy-selective yeast growth, with hygromycin B added to the indicated concentration, or synthetic complete dextrose media (SCD) for auxotrophy-selective growth, both supplemented with $2 \%$ glucose [41]. Yeast media components were supplied by Fisher Scientific and Formedium (Norfolk, UK).

\subsection{Yeast Transformations}

All yeast transformations were performed using the lithium acetate method with a 20 min heat shock at $42{ }^{\circ} \mathrm{C}$ prior to plating on appropriate selective media [42].

\subsection{Genomic and Plasmid DNA Isolation}

Genomic DNA was isolated for PCRTag analysis using phenol-chloroform extraction [43]. Plasmids were isolated from bacterial hosts using the QIAprep spin Miniprep Kit (Qiagen) (Hilden, Germany).

\subsection{Double Deletion Strains}

Double deletion strains were constructed starting from a single deletion strain derived from KO yeast collections [44] either BY4742 or BY4741. The URA3 deletion cassette was generated by PCR and transformed into the respective single deletion strain. The target gene was deleted using homologues recombination machinery. The confirmation PCR was performed to verify upstream and downstream chromosomal integration sites. 


\subsection{SCRaMbLE Workflow}

A single colony of the semi-synthetic synII strain (YCy1188) bearing pSCW11-creEBD was inoculated in SCD-His and grown overnight at $30^{\circ} \mathrm{C}$. The overnight culture was used to inoculate $10 \mathrm{ml}$ of SCD-His to an $\mathrm{OD}_{600}$ of 0.1. $\beta$-estradiol (Sigma Aldrich) (Munich, Germany).was added to a final concentration of $1 \mu \mathrm{M}$ to induce Cre expression and SCRaMbLE in cells. Cultures were grown for $24 \mathrm{~h}$, shaking at $30^{\circ} \mathrm{C}$. Cultures were back diluted to $0.1 \mathrm{OD}_{600}$ and $50 \mu \mathrm{L}$ aliquots were plated onto YPD plates containing different concentrations of hygromycin B (Thermo) (Dreieich, Germany) without selection for pSCW11-cre-EBD. Plates were incubated at $30{ }^{\circ} \mathrm{C}$ for $2-3$ days in order to select for hygromycin B resistant strains.

\subsection{Post-SCRaMbLE Selection for Hygromycin B Resistance}

Following SCRaMbLE of synII (YCy1188), single colonies of resistant strains were selected for further characterisation based on the colony size and ability to grow at $200 \mu \mathrm{g} / \mathrm{mL}$ of hygromycin B. Then, a spot test assay was performed on YPD plates containing different concentrations of hygromycin B. For this, each strain was inoculated into $5 \mathrm{~mL}$ of YPD medium for $24 \mathrm{~h}$, then re-inoculated to obtain a serial dilution starting with $0.1 \mathrm{OD}_{600}$.

\subsection{PCRTag Analysis of HYG2.1 to HYG2.5 Strains}

For each genomic DNA sample, the following master mix was prepared: $6.25 \mu \mathrm{L}$ of DreamTaq Green PCR Master Mix (2X) (ThermoFisher Scientific), $2.5 \mu \mathrm{L}$ of genomic DNA $(20 \mathrm{ng} / \mu \mathrm{L})$ and $2.25 \mu \mathrm{L}$ of sterile distilled water. $11 \mu \mathrm{L}$ of the master mix was aliquoted into each well of a 96-well PCR plate and $1.5 \mu \mathrm{L}$ of pre-mixed forward and reverse PCRTag primers $(10 \mathrm{mM})$ were added. The PCR thermal-cycler program was as follows: $94{ }^{\circ} \mathrm{C} / 3$ min, 30 cycles of $\left(94{ }^{\circ} \mathrm{C} / 30 \mathrm{~s}, 60^{\circ} \mathrm{C} / 30 \mathrm{~s}, 72{ }^{\circ} \mathrm{C} / 30 \mathrm{~s}\right)$, and a final extension of $72{ }^{\circ} \mathrm{C} / 7 \mathrm{~min}$. PCR samples were loaded directly onto a $1 \%$ agarose gel for electrophoresis and bands were visualized using a ChemiDoc ${ }^{\mathrm{TM}} \mathrm{XRS}+$ System (Bio-Rad). The presence or the absence of the amplicon was assessed visually.

\subsection{Nanopore Sequencing}

High quality DNA extraction was performed according to a modified Qiagen Genomictip 100/g protocol with the Qiagen Genomic Buffer kit. DNA quality was assessed via gel-electrophoresis, NanoDrop ${ }^{\mathrm{TM}} 2000$ Spectrophotometer and Qubit 4 Fluorometer using dsDNA BR reagents. Library preparation was performed using the SQK-LSK108 library kit with the Native Barcoding kits EXP-NDB104 and EXP-NDB113. Kits were used largely according to the manufacturers' guidelines, however input DNA was not sheared and the starting DNA concentration was increased 5-fold to match the molarity expected in the protocol. This was based on our experience with PFGE analysis of similarly prepared genomic DNA (data not shown). Sequencing was performed on a MinION Mk1B device using FLO-MIN106D with R9.4.1 chemistry. DNA sequencing was performed for 48 hours using the software MinKNOW v19.05.0.

Base calling and demultiplexing was performed locally using Guppy software (v3.1.5). Data obtained were mapped against the reference genome of BY4741 chrII::synII using minimap2 (v2.17) [45] and NGMLR (v0.2.7) [46]. Rearrangements in the NGMLR mapping data were called using Sniffles (v1.0.11) [46] with a threshold of $\geq 10$ reads confirming the rearrangement. De novo genome assembly of each strain was performed using Canu (v1.8) [47]. SCRaMbLE rearrangements in synII were evaluated and confirmed by comparing the mapping and variant calling data from Sniffles with the de novo assembly obtained by Canu [47].

\section{Conclusions and Perspectives}

Past studies have shown that $\mathrm{SCRaMbLE}$ is a powerful yet versatile tool that can be used to evolve the genotype of the synthetic strains to tolerate increased stressors, such as alkaline condition, high temperature and ethanol $[9,10]$. In this study, we show that the 
current SCRaMbLE system in Sc2.0 allowed us to generate gene rearrangements/structural variations in synthetic yeast containing synthetic yeast chromosome II that give rise to improved hygromycin B resistance. The function of SCRaMbLE is analogous to a combinatorial black-box. Therefore, improved strains can be generated without prior knowledge by simply shuffling genes in the synthetic chromosome(s). Desired phenotypes are subsequently selected from the enormous pool of genotypes produced. This stochastic process sheds new light on the possibilities of large-scale studies that were previously infeasible when using pools of randomly mutagenised yeasts, or via synthetic lethal array technology.

Despite our effort, the mechanism behind the higher hygromycin B resistance generated remains elusive. Gene deletions identified in the resistant SCRaMbLEd strains led us to assess the hygromycin B resistance of the corresponding single knockout strains. We were able to identify two uncharacterized genes, YBR219C and YBR220C, deletion of which led to slight, previously unreported improvements in the hygromycin $B$ resistance level. However, the individual gene deletions could not reach the resistance level conferred by complex structural variations generated by SCRaMbLE. The nanopore sequencing technology was used to deconvolute the structural rearrangements of the SCRaMbLEd genomes. However, the nanopore sequencing coverage within this study is not sufficient to confidently detect individual SNPs, but we have in the past performed comprehensive SCRaMbLE genome sequencing with Illumina technology and found the frequency of SNPs in these genomes are on par with their parental un-SCRaMbLEd [4]. Additionally, we have included a parental unSCRaMbLEd strain (synII) in this experiment. After selection, no resistance phenotype of the unSCRamBLEd synII could be obtained. This suggests it is unlikely that SNPs play are the causative of the observed HygB resistance phenotype. Nevertheless, increasing nanopore sequencing coverage or performing short read sequencing would be implemented in future characterization of SCRaMbLEd synthetic yeast strains.

Future in-depth investigations such as transcriptome profiling of multiple different resistant strains may indicate common patterns that could potentially point to the origin of the resistance.

Interestingly while we were attempting to inspect the capability of SCRaMbLE, we observed that the deletion of $A G P 2$ rendered the strain sensitive to hygromycin $\mathrm{B}$, instead of becoming more resistant as has been reported previously [27]. Conversely, FES1 knockout elicited the expected improvement in hygromycin B resistance [28]. Furthermore, we also investigated the reason why FES1 deletion was not observed via SCRaMbLE. FES1 is coupled to the essential gene EXO84 as part of an essential raft. Deletion of FES1 would be possible if the neighbouring essential gene EXO84 in the essential raft is moved or integrated into a different locus in the genome. Alternatively, inserting an additional loxP downstream to the FES1 gene using CRISPR would bypass this problem. This will likely further improve the resistance phenotype of the synthetic yeast after SCRaMbLE.

Notwithstanding, facing this challenge could present a new opportunity to improve SCRaMbLE. Systematic single deletions are routinely done without the necessity of synthetic chromosomes or SCRaMbLE. The SCRaMbLE system is, however, ideally poised to allow the investigation of more complex variations that are impossible through systematic mutations. Here we showed how SCRaMbLE was able to detect other complex genome modifications that yeast can develop for hygromycin B resistance. This may also put forward an alternative strategy, where the known resistance genes are deliberately coupled to essential genes so that effects of other genes can be assessed. Whether by itself or with the help of customised modifications, SCRaMbLE can help to anticipate the emergence of antibiotic resistance.

Supplying essential genes on a separate chromosome could be an alternative strategy to increase the deletion power of SCRaMbLE and increase the plasticity of the synthetic genome to obtain more desirable phenotypes. This strategy has been demonstrated by a recent paper [40] and proposed in the highly anticipated Sc3.0 project. It is also worth mentioning that this study was performed using only one synthetic chromosome. By using a fully synthetic strain and/or the improved SCRaMbLE system in Sc3.0, we should be 
able to obtain even more complex SCRaMbLE results and variants with higher antibiotic resistance.

Supplementary Materials: The following are available online at https:/ / www.mdpi.com/2306-535 $4 / 8 / 3 / 42 / s 1$.

Author Contributions: The study was conceived and conducted by J.Y.O., R.S., Y.C. and J.D. Y.C. and J.D. constructed the synthetic chromosomes and obtained funding for this project. M.M. and D.S. performed and analysed the nanopore sequencing. J.Y.O., R.S., D.S. and Y.C. wrote the manuscript. All authors edited and approved the final manuscript. All authors have read and agreed to the published version of the manuscript.

Funding: This work is supported by BBSRC Grant (R121730) and Volkswagen Foundation the "Life? Initiative" Grant (Ref. 94 771) both to YC. JD is supported by a Royal Society Newton Advanced Fellowship (NAF $\backslash$ R2 $\backslash 180590$ ) hosted by YC. JYO was supported with a MyBrainSc scholarship from the Ministry of Higher Education of Malaysia.

Data Availability Statement: All strains and vectors used in this study are available and can be obtained from the lab of Yizhi Cai (https: / / www.cailab.org, accessed on 17 March 2021). Any project details can also be provided upon request. All sequencing data are available on NCBI-Sequence Read Archive (SRA) under the BioProject ID PRJNA683650. The BioSample accessions are SAMN17034049, SAMN17034050, SAMN17034051 and SAMN17034052.

Acknowledgments: We thank Joshua James for circumstantial proof reading of the manuscript.

Conflicts of Interest: The authors declare no conflict of interest.

\section{References}

1. Gibson, D.G.; Benders, G.A.; Andrews-Pfannkoch, C.; Denisova, E.A.; Baden-Tillson, H.; Zaveri, J.; Stockwell, T.B.; Brownley, A.; Thomas, D.W.; Algire, M.A.; et al. Complete chemical synthesis, assembly, and cloning of a Mycoplasma genitalium genome. Science 2008, 319, 1215-1220. [CrossRef] [PubMed]

2. Richardson, S.M.; Mitchell, L.A.; Stracquadanio, G.; Yang, K.; Dymond, J.S.; DiCarlo, J.E.; Lee, D.; Huang, C.L.V.; Chandrasegaran, S.; Cai, Y.; et al. Design of a synthetic yeast genome. Science 2017, 355, 1040-1044. [CrossRef] [PubMed]

3. Dymond, J.S.; Richardson, S.M.; Coombes, C.E.; Babatz, T.; Muller, H.; Annaluru, N.; Blake, W.J.; Schwerzmann, J.W.; Dai, J.; Lindstrom, D.L.; et al. Synthetic chromosome arms function in yeast and generate phenotypic diversity by design. Nature 2011, 477, 471. [CrossRef]

4. Shen, Y.; Stracquadanio, G.; Wang, Y.; Yang, K.; Mitchell, L.A.; Xue, Y.; Cai, Y.; Chen, T.; Dymond, J.S.; Kang, K.; et al. SCRaMbLE generates designed combinatorial stochastic diversity in synthetic chromosomes. Genome Res. 2016, 26, 36-49. [CrossRef]

5. Blount, B.A.; Gowers, G.O.F.; Ho, J.C.H.; Ledesma-Amaro, R.; Jovicevic, D.; McKiernan, R.M.; Xie, Z.X.; Li, B.Z.; Yuan, Y.J.; Ellis, T. Rapid host strain improvement by in vivo rearrangement of a synthetic yeast chromosome. Nat. Commun. 2018, 9, 1932. [CrossRef]

6. Liu, W.; Luo, Z.; Wang, Y.; Pham, N.T.; Tuck, L.; Pérez-Pi, I.; Liu, L.; Shen, Y.; French, C.; Auer, M.; et al. Rapid pathway prototyping and engineering using in vitro and in vivo synthetic genome SCRaMbLE-in methods. Nat. Commun. 2018, 9, 1936. [CrossRef] [PubMed]

7. Jia, B.; Wu, Y.; Li, B.-Z.; Mitchell, L.A.; Liu, H.; Pan, S.; Wang, J.; Zhang, H.-R.; Jia, N.; Li, B.; et al. Precise control of SCRaMbLE in synthetic haploid and diploid yeast. Nat. Commun. 2018, 9, 1933. [CrossRef]

8. Gowers, G.O.F.; Chee, S.M.; Bell, D.; Suckling, L.; Kern, M.; Tew, D.; McClymont, D.W.; Ellis, T. Improved betulinic acid biosynthesis using synthetic yeast chromosome recombination and semi-automated rapid LC-MS screening. Nat. Commun. 2020, 11, 868. [CrossRef]

9. Luo, Z.; Wang, L.; Wang, Y.; Zhang, W.; Guo, Y.; Shen, Y.; Jiang, L.; Wu, Q.; Zhang, C.; Cai, Y.; et al. Identifying and characterizing SCRaMbLEd synthetic yeast using ReSCuES. Nat. Commun. 2018, 9, 1930. [CrossRef] [PubMed]

10. Ma, L.; Li, Y.; Chen, X.; Ding, M.; Wu, Y.; Yuan, Y.-J. SCRaMbLE generates evolved yeasts with increased alkali tolerance. Microb. Cell Factories 2019, 18, 52. [CrossRef]

11. Bongomin, F.; Gago, S.; Oladele, R.O.; Denning, D.W. Global and multi-national prevalence of fungal diseases-estimate precision. J. Fungi. 2017, 3, 57. [CrossRef]

12. Mann, R.L.; Bromer, W.W. The isolation of a second antibiotic from Streptomyces hygroscopicus. J. Am. Chem. Soc. 1958, 80, 2714-2716. [CrossRef]

13. Borovinskaya, M.A.; Shoji, S.; Fredrick, K.; Cate, J.H.D. Structural basis for hygromycin B inhibition of protein biosynthesis. RNA 2008, 14, 1590-1599. [CrossRef] [PubMed]

14. Zierhut, G.; Piepersberg, W.; Bock, A. Comparative analysis of the effect of aminoglycosides on bacterial protein synthesis in vitro. Eur. J. Biochem. 1979, 98, 577-583. [CrossRef] [PubMed] 
15. Brodersen, D.E.; Clemons, W.M., Jr.; Carter, A.P.; Morgan-Warren, R.J.; Wimberly, B.T.; Ramakrishnan, V. The structural basis for the action of the antibiotics tetracycline, pactamycin, and hygromycin B on the 30S ribosomal subunit. Cell 2000, 103, 1143-1154. [CrossRef]

16. Moazed, D.; Noller, H.F. Interaction of antibiotics with functional sites in 16S ribosomal RNA. Nature 1987, 327, 389-394. [CrossRef] [PubMed]

17. González, A.; Jiménez, A.; Vázquez, D.; Davies, J.E.; Schindler, D. Studies on the mode of action of hygromycin B, an inhibitor of translocation in eukaryotes. Biochim. Biophys. Acta (Bba) Nucleic Acids Protein Synth. 1978, 521, 459-469. [CrossRef]

18. Leboul, J.; Davies, J. Enzymatic modification of hygromycin B in Streptomyces hygroscopicus. J. Antibiot. 1982, 35, 527-528. [CrossRef]

19. Pardo, J.M.; Malpartida, F.; Rico, M.; Jimenez, A. Biochemical basis of resistance to hygromycin B in Streptomyces hygroscopicus-the producing organism. J. Gen. Microbiol. 1985, 131, 1289-1298. [CrossRef] [PubMed]

20. Zalacain, M.; Pardo, J.M.; Jimenez, A. Purification and characterization of a hygromycin B phosphotransferase from Streptomyces hygroscopicus. Eur. J. Biochem. 1987, 162, 419-422. [CrossRef]

21. Rao, R.N.; Allen, N.E.; Hobbs, J.N., Jr.; Alborn, W.E., Jr.; Kirst, H.A.; Paschal, J.W. Genetic and enzymatic basis of hygromycin B resistance in Escherichia coli. Antimicrob. Agents Chemother. 1983, 24, 689-695. [CrossRef]

22. Pfister, P.; Risch, M.; Brodersen, D.E.; Böttger, E.C. Role of $16 \mathrm{~S}$ rRNA helix 44 in ribosomal resistance to hygromycin B. Antimicrob. Agents Chemother. 2003, 47, 1496-1502. [CrossRef]

23. De Stasio, E.A.; Dahlberg, A.E. Effects of mutagenesis of a conserved base-paired site near the decoding region of Escherichia coli 16 S ribosomal RNA. J. Mol. Biol. 1990, 212, 127-133. [CrossRef]

24. Spangler, E.A.; Blackburn, E.H. The nucleotide sequence of the $17 \mathrm{~S}$ ribosomal RNA gene of Tetrahymena thermophila and the identification of point mutations resulting in resistance to the antibiotics paromomycin and hygromycin. J. Biol. Chem. 1985, 260, 6334-6340. [CrossRef]

25. Gritz, L.; Davies, J. Plasmid-encoded hygromycin B resistance: The sequence of hygromycin B phosphotransferase gene and its expression in Escherichia coli and Saccharomyces cerevisiae. Gene 1983, 25, 179-188. [CrossRef]

26. Kaster, K.R.; Burgett, S.G.; Ingolia, T.D. Hygromycin B resistance as dominant selectable marker in yeast. Curr. Genet. 1984, 8 , 353-358. [CrossRef] [PubMed]

27. Aouida, M.; Khodami-Pour, A.; Ramotar, D. Novel role for the Saccharomyces cerevisiae oligopeptide transporter Opt2 in drug detoxification. Biochem. Cell Biol. Biochim. Biol. Cell. 2009, 87, 653-661. [CrossRef] [PubMed]

28. Barreto, L.; Canadell, D.; Petrezsélyová, S.; Navarrete, C.; Marešová, L.; Peréz-Valle, J.; Herrera, R.; Olier, I.; Giraldo, J.; Sychrová, H.; et al. A genomewide screen for tolerance to cationic drugs reveals genes important for potassium homeostasis in Saccharomyces cerevisiae. Eukaryot. Cell 2011, 10, 1241-1250. [CrossRef] [PubMed]

29. Lussier, M.; White, A.M.; Sheraton, J.; di Paolo, T.; Treadwell, J.; Southard, S.B.; Horenstein, C.I.; Chen-Weiner, J.; Ram, A.F.; Kapteyn, J.C.; et al. Large scale identification of genes involved in cell surface biosynthesis and architecture in Saccharomyces cerevisiae. Genetics 1997, 147, 435-450. [CrossRef]

30. Chawla, B.; Jhingran, A.; Panigrahi, A.; Stuart, K.D.; Madhubala, R. Paromomycin affects translation and vesicle-mediated trafficking as revealed by proteomics of paromomycin -susceptible -resistant Leishmania donovani. PLoS ONE 2011, 6, e26660. [CrossRef]

31. Lindstrom, D.L.; Gottschling, D.E. The mother enrichment program: A genetic system for facile replicative life span analysis in Saccharomyces cerevisiae. Genetics 2009, 183, 413-422. [CrossRef] [PubMed]

32. Ghosh, S.K.; Poddar, A.; Hajra, S.; Sanyal, K.; Sinha, P. The IML3/MCM19 gene of Saccharomyces cerevisiae is required for a kinetochore-related process during chromosome segregation. Mol. Genet. Genom. 2001, 265, 249-257. [CrossRef]

33. Michelot, A.; Grassart, A.; Okreglak, V.; Costanzo, M.; Boone, C.; Drubin, D.G. Actin Filament Elongation in Arp2/3-Derived Networks Is Controlled by Three Distinct Mechanisms. Dev. Cell 2013, 24, 182-195. [CrossRef] [PubMed]

34. Giaever, G.; Chu, A.M.; Ni, L.; Connelly, C.; Riles, L.; Véronneau, S.; Dow, S.; Lucau-Danila, A.; Anderson, K.; André, B.; et al. Functional profiling of the Saccharomyces cerevisiae genome. Nature 2002, 418, 387-391. [CrossRef] [PubMed]

35. Gowda, N.K.C.; Kandasamy, G.; Froehlich, M.S.; Dohmen, R.J.; Andréasson, C. Hsp70 nucleotide exchange factor Fes1 is essential for ubiquitin-dependent degradation of misfolded cytosolic proteins. Proc. Natl. Acad. Sci. USA 2013, 110, 5975-5980. [CrossRef]

36. Gowda, N.K.C.; Kaimal, J.M.; Masser, A.E.; Kang, W.; Friedländer, M.R.; Andréasson, C. Cytosolic splice isoform of Hsp70 nucleotide exchange factor Fes1 is required for the degradation of misfolded proteins in yeast. Mol. Biol. Cell 2016, 27, 1210-1219. [CrossRef]

37. Aouida, M.; Page, N.; Leduc, A.; Peter, M.; Ramotar, D. A genome-wide screen in Saccharomyces cerevisiae reveals altered transport as a mechanism of resistance to the anticancer drug bleomycin. Cancer Res 2004, 64, 1102-1109. [CrossRef]

38. Winston, F.; Dollard, C.; Ricupero-Hovasse, S.L. Construction of a set of convenient saccharomyces cerevisiae strains that are isogenic to S288C. Yeast 1995, 11, 53-55. [CrossRef]

39. Baker Brachmann, C.; Davies, A.; Cost, G.J.; Caputo, E.; Li, J.; Hieter, P.; Boeke, J.D. Designer deletion strains derived from Saccharomyces cerevisiae S288C: A useful set of strains and plasmids for PCR-mediated gene disruption and other applications. Yeast 1998, 14, 115-132. [CrossRef]

40. Guthrie, C.; Fink, G.R. Guide to yeast genetics and molecular biology. Methods Enzym. 1991, 194, 1-863. 
41. Gietz, R.D.; Schiestl, R.H.; Willems, A.R.; Woods, R.A. Studies on the transformation of intact yeast cells by the LiAc/SSDNA/PEG procedure. Yeast 1995, 11, 355-360. [CrossRef]

42. Hoffman, C.S.; Winston, F. A ten-minute DNA preparation from yeast efficiently releases autonomous plasmids for transformation of Escherichia coli. Gene 1987, 57, 267-272. [CrossRef]

43. Li, H. Minimap2: Pairwise alignment for nucleotide sequences. Bioinformatics 2018, 34, 3094-3100. [CrossRef]

44. Sedlazeck, F.J.; Rescheneder, P.; Smolka, M.; Fang, H.; Nattestad, M.; von Haeseler, A.; Schatz, M.C. Accurate detection of complex structural variations using single-molecule sequencing. Nat. Methods 2018, 15, 461-468. [CrossRef] [PubMed]

45. Koren, S.; Walenz, B.P.; Berlin, K.; Miller, J.R.; Bergman, N.H.; Phillippy, A.M. Canu: Scalable and accurate long-read assembly via adaptive k-mer weighting and repeat separation. Genome Res. 2017, 27, 722-736. [CrossRef] [PubMed]

46. Wang, P.; Xu, H.; Li, H.; Chen, H.; Zhou, S.; Tian, F.; Li, B.-Z.; Bo, X.; Wu, Y.; Yuan, Y.-J. SCRaMbLEing of a synthetic yeast chromosome with clustered essential genes reveals synthetic lethal interactions. ACS Synth. Biol. 2020, 9, 1181-1189. [CrossRef] [PubMed]

47. Dai, J.; Boeke, J.D.; Luo, Z.; Jiang, S.; Cai, Y. Sc3.0: Revamping and minimizing the yeast genome. Genome Biol. 2020, 21, 205. [CrossRef] 